\title{
FRACTIONAL REVIVAL OF THRESHOLD GRAPHS UNDER LAPLACIAN DYNAMICS
}

\author{
Steve Kirkland and XiaOhong Zhang \\ Department of Mathematics, University of Manitoba \\ Winnipeg, MB, Canada R3T 2N2 \\ e-mail: stephen.kirkland@umanitoba.ca \\ zhangx $42 @$ myumanitoba.ca
}

This paper is dedicated to the memory of Slobodan Simić.

\begin{abstract}
We consider Laplacian fractional revival between two vertices of a graph $X$. Assume that it occurs at time $\tau$ between vertices 1 and 2 . We prove that for the spectral decomposition $L=\sum_{r=0}^{q} \theta_{r} E_{r}$ of the Laplacian matrix $L$ of $X$, for each $r=0,1, \ldots, q$, either $E_{r} e_{1}=E_{r} e_{2}$, or $E_{r} e_{1}=-E_{r} e_{2}$, depending on whether $e^{i \tau \theta_{r}}$ equals to 1 or not. That is to say, vertices 1 and 2 are strongly cospectral with respect to $L$. We give a characterization of the parameters of threshold graphs that allow for Laplacian fractional revival between two vertices; those graphs can be used to generate more graphs with Laplacian fractional revival. We also characterize threshold graphs that admit Laplacian fractional revival within a subset of more than two vertices. Throughout we rely on techniques from spectral graph theory.

Keywords: Laplacian matrix, spectral decomposition, quantum information transfer, fractional revival.
\end{abstract}

2010 Mathematics Subject Classification: 05C50, 15A18, 81P45.

\section{REFERENCES}

[1] R. Alvir, S. Dever, B. Lovitz, J. Myer, C. Tamon, Y. Xu and H. Zhan, Perfect state transfer in Laplacian quantum walk, J. Algebraic Combin. 43 (2016) 801-826. doi:10.1007/s10801-015-0642-x

[2] R.J. Angels-Canul, E. Norton, M. Opperman, C. Paribello, M. Russell and C. Tamon, Quantum perfect state transfer on weighted join graphs, Int. J. Quantum Inf. 07 (2009) 1429-1445. doi:10.1142/S0219749909006103 
[3] R. Bachman, E. Fredette, J. Fuller, M. Landry, M. Opperman, C. Tamon and A. Tollefson, Perfect state transfer on quotient graphs, Quantum Inf. Comput. 12 (2012) 293-313.

[4] M. Bašić, M.D. Petković and D. Stevanović, Perfect state transfer in integral circulant graphs, Appl. Math. Lett. 22 (2009) 1117-1121. doi:10.1016/j.aml.2008.11.005

[5] P.-A. Bernard, A. Chan, É. Loranger, C. Tamon and L. Vinet, A graph with fractional revival, Phys. Lett. A 382 (2018) 259-264. doi:10.1016/j.physleta.2017.12.001

[6] D.M. Cardoso, C. Delorme and P. Rama, Laplacian eigenvectors and eigenvalues and almost equitable partitions, European J. Combin. 28 (2007) 665-673. doi:10.1016/j.ejc.2005.03.006

[7] A. Chan, G. Coutinho, C. Tamon, L. Vinet and H. Zhan, Quantum fractional revival on graphs (2018), manuscript. http://arxiv.org:1801.09654, 2018

[8] A. Chan and J. Teitelbaum, personal communication, (2018).

[9] W.-C. Cheung and C. Godsil, Perfect state transfer in cubelike graphs, Linear Algebra Appl. 435 (2011) 2468-2474. doi:10.1016/j.laa.2011.04.022

[10] M. Christandl, N. Datta, T.C. Dorlas, A. Ekert, A. Kay and A.J. Landahl, Perfect transfer of arbitrary states in quantum spin networks, Phys. Rev. A 71 (2005) 032312 . doi:10.1103/PhysRevA.71.032312

[11] V.X. Genest, L. Vinet and A. Zhedanov, Quantum spin chains with fractional revival, Ann. Physics 371 (2016) 348-367. doi:10.1016/j.aop.2016.05.009

[12] C. Godsil, Algebraic Combinatorics (Chapman \& Hall, New York, 1993).

[13] C. Godsil, State transfer on graphs, Discrete Math. 312 (2012) 129-147. doi:10.1016/j.disc.2011.06.032

[14] C. Godsil, When can perfect state transfer occur?, Electron. J. Linear Algebra 23 (2012) 877-890. doi:10.13001/1081-3810.1563

[15] A. Kay, A review of perfect state transfer and its application as a constructive tool, Int. J. Quantum Inf. 8641 (2010) 641-676. doi:10.1142/S0219749910006514

[16] M. Kempton, G. Lippner and S.-T. Yau, Perfect state transfer on graphs with a potential, Quantum Inf. Comput. 17 (2017) 303-327. doi:10.1103/PhysRevA.83.012310

[17] S. Kirkland and S. Severini, Spin-system dynamics and fault detection in threshold networks, Phys. Rev. A 83 (2011) 012310. 
[18] N.V.R. Mahadev and U.N. Peled, Threshold Graphs and Related Topics (NorthHolland Publishing Co., Amsterdam, 1995).

Received 9 November 2018

Revised 2 May 2019

Accepted 2 May 2019 